\title{
Pediatric palliative care: how early should be planned?
}

\begin{abstract}
Pediatric palliative care should be provided to every child at the diagnosis of a lifethreatening condition. In addition to respecting the distress of bad news and the family mechanisms of coping, it is vital to define a realistic and adequate strategy of care. Considering the disease's trajectory, an anticipatory guide of care can provide a unique instrument to prepare parents for decisions in unexpected situations. We report a clinical situation of end-of-life care, reflecting on some challenges of pediatric palliative care.
\end{abstract}

Keywords: pediatric palliative care, cerebral palsy, withholding treatment, end-oflife
Volume 2 Issue 5 - 2018

\author{
Inês Viegas, Maria João Palaré, Maria-Céu \\ Machado \\ Departamento de Pediatria, Hospital Santa Maria, Centro \\ Académico de Medicina de Lisboa, Portugal
}

Correspondence: Inês Viegas, Department of Pediatrics, Hospital de Santa Maria, Centro Académico de Medicina de Lisboa, 1649-028, Lisbon, Portugal, Tel +35 I912308203,

Email ines.viggas@campus.ul.pt

Received: September 18, 2018 | Published: November 05, 2018
Abbreviations: PICU, pediatric intensive care unit; DNR, do not resuscitate; cPAP, continuous positive airway pressure

\section{Introduction}

Dealing with children suffering from complex chronic conditions has always been challenging to pediatricians and health care teams because of the poor prognosis it involves. Sometimes there is no possible cure neither an improvement in the quality of life can be considered.

All the pediatricians, whichever their subspecialty, provide unquestionable care to these children and their families but the pediatric palliative care team has a unique role in anticipating unexpected situations during the course of the plan of care. In fact, one of the aims of pediatric palliative care is to provide care across the age spectrum and life span, in a continuum of care. ${ }^{2}$

Children represent a highly susceptible group, both physically and socially. The ethical principle of autonomy is limited by age and psychomotor development and mostly held by parents or legal representatives, in the best interest of the child. The rights and limits of this autonomy vary considerably across the world and the legal aspects of each country have to be taken into account.

Withholding or withdrawing treatments and settling a do not resuscitate order in pediatrics are difficult limits of palliative care that should be considered by the parents and the team. The acceptance of the parents can help deal with disease exacerbations, acute failures, and sudden physical deterioration or even with death and mourning.

\section{Case report}

Rafael was a five year-old boy, first son of a young and nonconsanguine couple. Born preterm, at 28 weeks of gestation, he had multiple sequelae: grade III intraventricular hemorrhage complicated with hydrocephalus and a ventriculo-peritoneal shunt; multicystic encephalomalacia, hemispheric bilateral cerebelous atrophy and a sensorineural hearing loss. Later, he was diagnosed with severe cerebral palsy (spastic quadriplegia) and secondary epilepsy. As comorbidities, he had chronic pulmonary disease with multiple previous hospitalizations due to exacerbations, presenting with persistent hypoxemia and needing supplementary oxygen at home and sleep non-invasive ventilation with cPAP; he had a neurogenic bladder with a grade III vesicoureteral reflux and nephrolithiasis, which made him susceptible for recurrent urinary tract infections with multi resistant microorganisms, submitted thereafter to a vesicostomy. He was fed by a gastrostomy tube.

From birth until age 5, he had been hospitalized many times, seen in several different pediatric subspecialty appointments and admitted innumerous times to the emergency department with acute exacerbations or infectious intercurrences. Either way, their son made it until age 5, even if they knew prognosis has been poor since birth, giving parents hope that survival until latter age might be possible.

At age of 5 years old his psychomotor development was greatly delayed in all the field acquisitions. He could recognize familiar faces and smile at them, as well as say a few words like "mama".

During one of the respiratory insufficiency exacerbations in the context of Pseudomonas aeruginosa pneumonia, he was submitted to mechanical ventilation in the ICU. Facing the inability to extubate, the hypothesis of a tracheostomy was engaged.

The decision of whether to proceed to a last invasive procedure have aroused an ethical dilemma, both to the parents and the healthcare team. Would it be plausible to go forward with this treatment in a child with limited social relation and so many morbidities? Or would it be futile? Would this mean prolonged pain and suffer for both the child and the family? What are the acceptable boundaries of quality of life and where does stand life dignity? Were the parents' request enough to withhold medical care?

Faced with these many ethical dilemmas the multidisciplinary team met with the parents with the objective of reaching a deliberation. This process began first and foremost by presenting the facts. By describing and analyzing the clinical situation and reviewing what the parents already knew the team was able to lay out grounds of understanding and avoid errors in the appreciation of the clinical situation that would drag unnecessarily the decision of how to proceed.

It was also important to identify what values where important for the family and for the team to define the optimal course of action. 
Furthermore, the interveners quickly identified the two extreme courses of action, to refuse the tracheostomy and withhold any possible treatments or to proceed with the treatments and do everything to prolong the child's life.

Without ever being engaged to think about this moment by and with the health care team, the parents had discussed it amongst themselves and were resolved about their position. They didn't want to prolong his son's life with more suffering. In the face of a cardiorespiratory arrest, they've chosen a do not resuscitate (DNR) position.

Despite their resolution the parents had many questions about how to proceed and what was offered once the decision was made. By explaining the procedures done and what would be the next steps, assuring continuity of care, the team was able to implement a plan that would bring most comfort to all the parts involved. Shortly after, the child died in the ICU under sedoanalgesia.

End-of-life care included multidisciplinary team follow-up, minimal invasive comfort care, as feeding and pain prevention/control and other symptoms approach. Parents participated in the child care until his last minute of life; a time was provided for other relatives and significant others to say goodbye in a private environment; he passed away in his mother's lap with the father by their side.

In a post-mortem debriefing, the health care team reflected about the end-of-life care that was provided. In fact, they thought that care provided in this situation should have been anticipated in the child's plan of care, from birth to the diagnosis and the prognosis of this child, who faced a multiple complex disease situation. A pediatric palliative care team would have been of great value, collaborating with the multidisciplinary team of medical care. The death could have occurred at home or in a private room, if that was the parents' wish, instead of happening at the muddled PICU environment.

This clinical case makes us contemplate the value of life, the meaning of quality of life, the decision of when to withhold treatment (tracheostomy) and when to accept a do not resuscitate order. Preparing families of children with chronic complex diseases for exacerbations, that can result in premature death, should be part of the palliative care team work along the pathway, not only when this turns out to be true.

\section{Discussion}

This clinical case exemplifies many others of children with cerebral palsy, a non-progressive, irreversible disease where there is a high probability of premature death, as well as a compromised quality of life, both for the parents and the child. ${ }^{3}$ Children with cerebral palsy and other neurologic conditions benefit from being referred to palliative care as soon as diagnosis is made.

Pediatric palliative care should be started at the diagnosis of a lifethreatening disease and continue along with curative care. It should not be misinterpreted only as end-of-life care, which it includes, nor faced as precluding the chances of survival. When diagnosis is made in utero, it can even start in pre-natal period. Neonatologists have long been confronted with the difficulties of providing care in these situations and dealing with newborn's death. As the limit of gestational age viability decreases, neonatologists and pediatricians frequently face the dilemma of when to resuscitate as well as the long term sequelae of prematurity, where palliative care needs might be present.

In facing each situation, even in pediatric age, it is mandatory that the parents or provider become aware of the expectable trajectory; in fact, a guide of anticipatory management of care can improve the patient quality of life and help the parents deal with certain circumstances in the future. This includes identification of procedures or interventions that may be no longer necessary or adequate for each of the disease status. ${ }^{4}$

It is essential that parents and children get involved in planning end-of-life care, even regarding the location of death. Not every child has to die in the hospital nor at home, but when parents are called to plan that moment, there is an association with more home deaths. ${ }^{5}$ Choosing to die at home definitely doesn't mean that the child will have no support when facing deterioration. There should be support available to call the pediatric palliative care team or even the emergency number and support by health professionals who can provide symptom relief at home, without the stressful surrounding of PICU.

In Portugal there are few teams available that provide pediatric palliative care, resulting in many pediatric teams having to face many problems and ethical dilemmas. Oftentimes, the on-call teams have to inform and work with a family that is not fully aware of the prognosis of the disease, doesn't know what the options of treatment are and has many misconceptions about palliative care.

Therefore, when faced with complex situations pediatricians should consider opinions, values and beliefs of the family when planning a course of action, trying to support the patient and the family and striving to look for the best solution. It is also important to study such clinical cases afterwards, not only doing an ethical analysis of the problem but also contrasting the decision with the legal norm, to ensure that it does not trespass or violate the limits established by law.

Ethical and legal aspects of end-of-life care in pediatrics are complex and differ by legal statements of each country. Even though parents' choices are taken into best account in clinical decisions, physicians are limited by both legal and ethical considerations, standing in a demanding position. ${ }^{1}$

The rise of modern medicine and particularly pediatric care have prolonged life expectancy considerably, which represents an increasing number of pediatric patients living with chronic complex conditions. Many of these conditions are rare, which makes the prognosis difficult to predict. Actually, some of these children survive until adult age, with palliative needs supported by pediatric teams that try to provide the best care in transition to adulthood.

While dealing with such a wide age range, from pre-natal until adolescence, pediatric palliative teams face the challenge of adapting to each patient's physical, emotional and cognitive stage of development. Families must also be a priority, as they are affected as a whole, never forgetting siblings and grandparents.

Children with chronic complex conditions are frequently hospitalized for prolonged periods, although there is evidence that home based care can be more appropriated. ${ }^{6}$ The pediatric palliative care teams working at pediatric services must assure that children are receiving the education needed for their age during hospitalization, while keeping a close collaboration with primary care teams, arranging the best conditions for a timely discharge. Unfortunately, in Portugal there are few teams that provide care at home, most directed to adult care and with no experience in pediatrics.

Contrary to what happens in many other countries around the world, there are no hospices in Portugal. Frequently, the only way to help family caregivers to get the rest they need is to hospitalize the child. Hospices or other similar facilities outside the hospital are a huge need in pediatric palliative care in our country. 
In what concerns end-of-life, children tend to receive more aggressive care, when compared to adults. ${ }^{7}$ The support of a pediatric palliative care team and the existence of an anticipated plan of care can be useful in not pursuing futile interventions, but education of all professionals about palliative care seems central towards this objective.

Only recently is pediatric palliative care knowledge starting to be seen as a fundamental competence in pediatrics, at least at a basic level. ${ }^{8}$ In Portugal, older pediatricians have not received this education during their training. Education in palliative care medicine has not yet reached every healthcare professional, which represent a major obstacle.

Pediatric palliative care is relatively recent in the history of pediatrics. Pediatricians have long before faced the challenges of following children and families with chronic complex conditions, trying to address their medical, emotional and social needs. Nevertheless, pediatric palliative care teams, with their specialized skills, can provide an indispensable aid, collaborating with other pediatric subspecialties. Ultimately, we all have the same goal: to relieve suffer and to provide a better life for our children.

\section{Acknowledgements}

None.

\section{Conflict of interest}

Authors declare that there is no conflict of interest.

\section{References}

1. Leeuwenburgh-Pronk WG, Miller-Smith L, Forman V, et al. Are we allowed to discontinue medical treatment in this child? Pediatrics. 2015;135(3):545-549.

2. Section on Hospice and Palliative Medicine and Committee on Hospital Care. Pediatric Palliative Care and Hospice Care Commitments, Guidelines, and Recommendations. Pediatrics. 2013;132(5):966-972.

3. Hain R, Wallace A. Progress in palliative care for children in the UK. Paediatrics and Child Health. 2008;18(3):141-146.

4. Levine D, Lam C, Cunningham M, et al. Best practices for pediatric palliative cancer care: a primer for clinical providers. J Support Oncol. 2013;11(3):114-125.

5. Dussel V, Kreicbergs U, Hilden JM, et al. Looking beyond where children die: determinants and effects of planning a child's location of death. J Pain Symptom Manage. 2009;37(1):33-43.

6. Integrating palliative care and symptom relief into paediatrics: a WHO guide for health care planners, implementers and managers. Licence: CC BY-NC-SA.3.0.IGO, Geneva: World Health Organization; 2018.

7. International Children's Palliative Care Network (UK). What is Children's Palliative Care? ICPCN; 2015.

8. American Academy of Pediatrics. Committee on Bioethics and Committee on Hospital Care. Palliative care for children. Pediatrics. 2000;106(2 Pt 1):351-357. 